\title{
Propagation Characteristics of Bragg Fiber
}

\author{
Pratibha Tanwar \\ Dept. ECE, Krishna Institute of \\ Engineering Technology, \\ Ghaziabad, UP, India
}

\author{
Shalini Sharma \\ Dept. ECE, Krishna Institute of \\ Engineering Technology, \\ Ghaziabad, UP, India
}

\author{
Amit Kumar \\ Dept. ECE, Krishna Institute of \\ Engineering Technology, \\ Ghaziabad, UP, India
}

\begin{abstract}
The propagation characteristics of optical fiber play a very important role in the design of a fiber optic communication system. In this paper, we have analyzed the Propagation Characteristics of Bragg fiber using transfer matrix method and have also found the modal filed distribution of the fundamental mode of Bragg fiber. We haves worked on the MATLAB and used its optical waveguide tool box for generation our results.
\end{abstract}

\section{Keywords}

Waveguide; Optical fibers; Bragg fibers; Transfer electromagnetic waves; Transfer magnetic waves.

\section{INTRODUCTION}

Bragg fiber is a new class of fiber, consisting of a periodical cladding in which light confinement is done due to the circular Bragg reflection in contrast to total internal reflection in conventional fiber, were proposed by Yeh et al.[1].Bragg fiber provide many different possibilities that are difficult to attain in conventional fiber because of his different guiding mechanism due to which it has attracted considerable recent interest among researchers, one such possibilities are guiding light in air[2].Which leads to lower propagation loss and reduced the threshold for nonlinear effects. A Bragg fiber can also be deigned to support the fundamental mode i.e. is non degenerate $T E_{01}$ mode with azimuthal dependence. In comparison with the fundamental mode in conventional fiber i.e. always doubly degenerate, The guiding modes of Bragg fiber are truly single mode [3].Numerous numerical analysis have been done to analyze the properties of Bragg fiber [1], Yeh et al. analyzed Bragg fibers successfully using Transfer matrix formalism, which used four parameters to describe the solution of Maxwell's equation in each layer of Bragg fiber and dielectric layer was related via $4 \times 4$ matrix. Bragg fiber modes were considered as quasi mode whose propagation constant and field distribution were found by minimizing the radiation loss [1].The rising numerical error makes the field calculation very sensitive to the propagation constants. In [47], the periodical cladding layers of Bragg fiber were approximated by planar Bragg stack using asymptotic approximation of Bessel function; therefore Bloch theorem [8] can be used to obtain an analytical Eigen equation. In this paper we will present the design and analysis of Bragg fiber using analytical solutions for studying different modal parameters of fiber. Here we have mainly concentrated on the propagation characteristics of fiber and analyzed the propagation constant of the multi layered index guiding Bragg fiber

\section{THEORETICAL ANALYSIS}

In the design of Bragg fiber, alternate Cladding layers of high $\left(n_{1}\right)$ and low $\left(n_{2}\right)$ refractive index are arranged around the central core having $\left(n_{c}\right)$ as the refractive index. The refractive index distribution of this Bragg fiber is pictorially represented in the fig.1.We will use the Transfer matrix formalism to analyze the propagation constant of Bragg fiber.

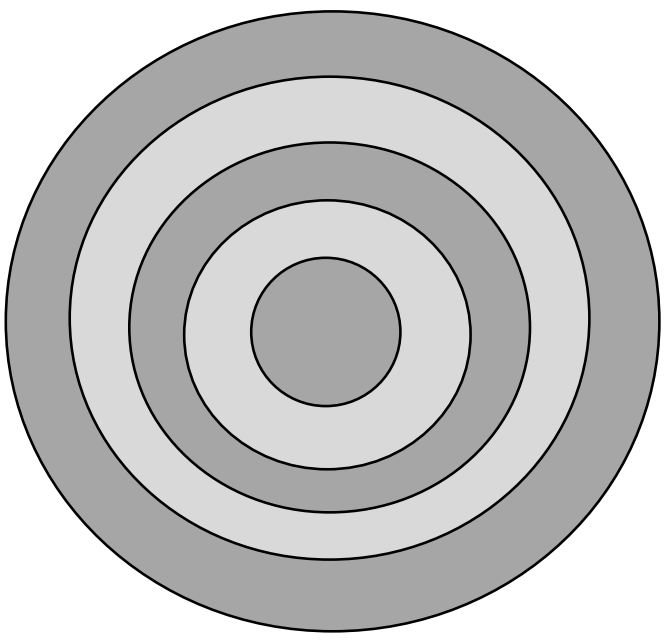

(a) Cross sectional view of Bragg Fiber

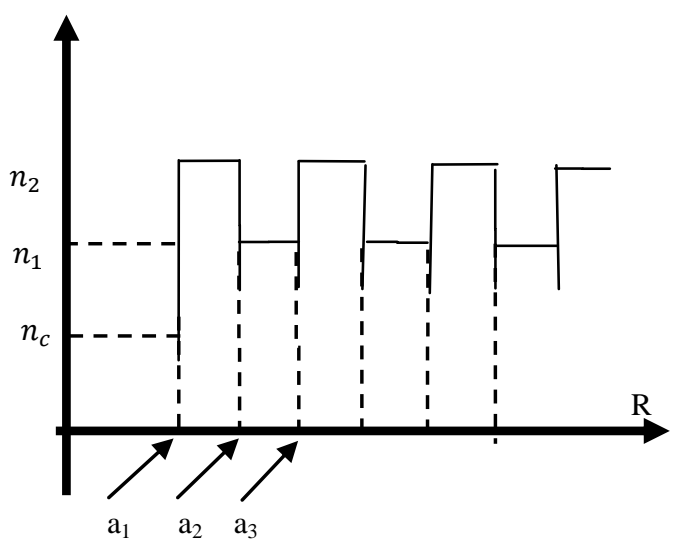

Fig 1- Refractive index profile of Bragg fiber

\subsection{Analytical Equations}

In each uniform cylindrical layer, the four transverse filed components $\left(E_{r}, E_{\varphi}, H_{r}\right.$ and $\left.H_{\varphi}\right)$ can be expressed as a linear combination of any two types of Bessel function[1].We have taken the z- direction as the direction of propagation, longitudinal component of magnetic or electric field will satisfy the Helmholtz equation[9] as given as

$$
\begin{gathered}
\frac{\partial^{2} H_{Z}\left(o r E_{Z}\right)}{\partial r^{2}}+\frac{1}{r} \frac{\partial H_{Z}\left(o r E_{Z}\right)}{\partial r}+\frac{1}{r^{2}} \frac{\partial^{2} H_{Z}\left(o r E_{Z}\right)}{\partial \phi^{2}} \\
+\left[k_{0}^{2} n^{2}(r)-\beta^{2}\right] H_{Z}\left(o r E_{Z}\right)=0
\end{gathered}
$$


The solution of this equation for different layers of fiber can be written as

$$
\begin{aligned}
& \psi(r, \varphi)=A_{1} Z_{l}\left(U_{1} r\right) \operatorname{Cos}(l \varphi) ; 0 \leq r \leq a_{1} \\
& \psi(r, \varphi)=A_{1} Z_{l}\left(U_{i} r\right)+B_{i} \widetilde{Z}_{l} \operatorname{Cos}(1 \varphi) ; \\
& a_{i} \leq r \leq a_{i+1} \\
& \psi(r, \varphi)=A_{1} \widetilde{Z}_{l}\left(U_{n} r\right) \operatorname{Cos}(1 \varphi) ; r>a_{n} \\
& U_{1}=a\left(k_{0}^{2} n_{1}^{2}-\beta^{2}\right)^{1 / 2} \\
& Z_{l}=J_{l}\left(U_{n}\right): \beta<n_{i} \\
& U_{i}=a\left(k_{0}^{2} n_{i}^{2}-\beta^{2}\right)^{1 / 2} \\
& Z_{l}=I_{l}\left(U_{n}\right): \beta>n_{i} \\
& U_{i}=a\left(\beta^{2}-k_{0}^{2} n_{i}^{2}\right)^{1 / 2} \\
& \widetilde{Z_{l}}=Y\left(U_{n}\right): \beta<n_{i} \\
& U_{n}=a\left(k_{0}^{2} n_{n}^{2}-\beta^{2}\right)^{1 / 2} \\
& \widetilde{Z_{l}}=K\left(U_{n}\right): \beta>n_{i}
\end{aligned}
$$

Now by using the matrix method and applying the continuity condition to $\psi$ and its normal derivate at each of the interfaces, we have obtained coefficient matrix given below,

Where $k_{i}$ and $n_{i}$ are transverse propagation constant and the refractive index of the $i^{\text {th }}$ layer respectively.

$k_{i}=k_{0} \sqrt{n_{i}^{2}-\beta^{2} / k_{0}^{2}}, k_{0}$ is the wave vector in the free space,

is the propagation constant and $\beta / k_{0}$ is the refractive index of the mode, A,B,C,D are the coefficients, and $m$ is the azimuthal modal number. When $\mathrm{m}=0$, the modes are decoupled into two polarizations, TE and TM modes. Now by resolving the determinant to zero we will obtain the mode effective indices and the corresponding filed distribution for different modes.

$\left[\begin{array}{l}E_{Z} \\ H_{\varphi} \\ H_{Z} \\ E_{\varphi}\end{array}\right]=$

$\left[\begin{array}{cccc}J_{l}\left(k_{i} a\right) & Y_{l}\left(k_{i} a\right) & 0 & 0 \\ \frac{\omega \epsilon_{i}}{\beta k_{i}} J^{\prime}{ }_{l}\left(k_{i} p\right) & \frac{\omega \epsilon_{i}}{\beta k_{i}} Y^{\prime}{ }_{l}\left(k_{i} p\right) & \frac{l}{k_{i}^{2} p} J_{l}\left(k_{i} p\right) & \frac{l}{k_{i}^{2} p} Y_{l}\left(k_{i} p\right) \\ 0 & 0 & J_{l}\left(k_{i} p\right) & Y_{l}\left(k_{i} p\right) \\ \frac{l}{k_{i}^{2} p} J_{l}\left(k_{i} p\right) & \frac{l}{k_{i}^{2} p} Y_{l}\left(k_{i} p\right) & \frac{\omega \mu_{i}}{\beta k_{i}} J^{\prime}{ }_{l}\left(k_{i} p\right) & \frac{\omega \mu_{i}}{\beta k_{i}} Y^{\prime}{ }_{l}\left(k_{i} p\right)\end{array}\right]\left[\begin{array}{c}A \\ B \\ C \\ D\end{array}\right]$

\section{RESULT AND DISCUSSION}

We have calculated the fundamental mode i.e. $L P_{01}$ mode filed distribution along with the structural parameters and the mode effective indices, in Fig-2 (a),(b) moreover we have also calculated the variation of " $b$ " the normalized propagation constant with respect to the normalized frequency "v" which is known as dispersion curve in Fig-3.where we have taken $\mathrm{a}=0.8335, \quad n_{\text {core }}=1.462420, \quad n_{g}=1.457420$ $n_{\text {eff } 01}=1.4622 \quad n_{\text {eff } 02}=1.4612 \quad n_{\text {eff } 03}=$ 1.4594 and get the dispersion curve.
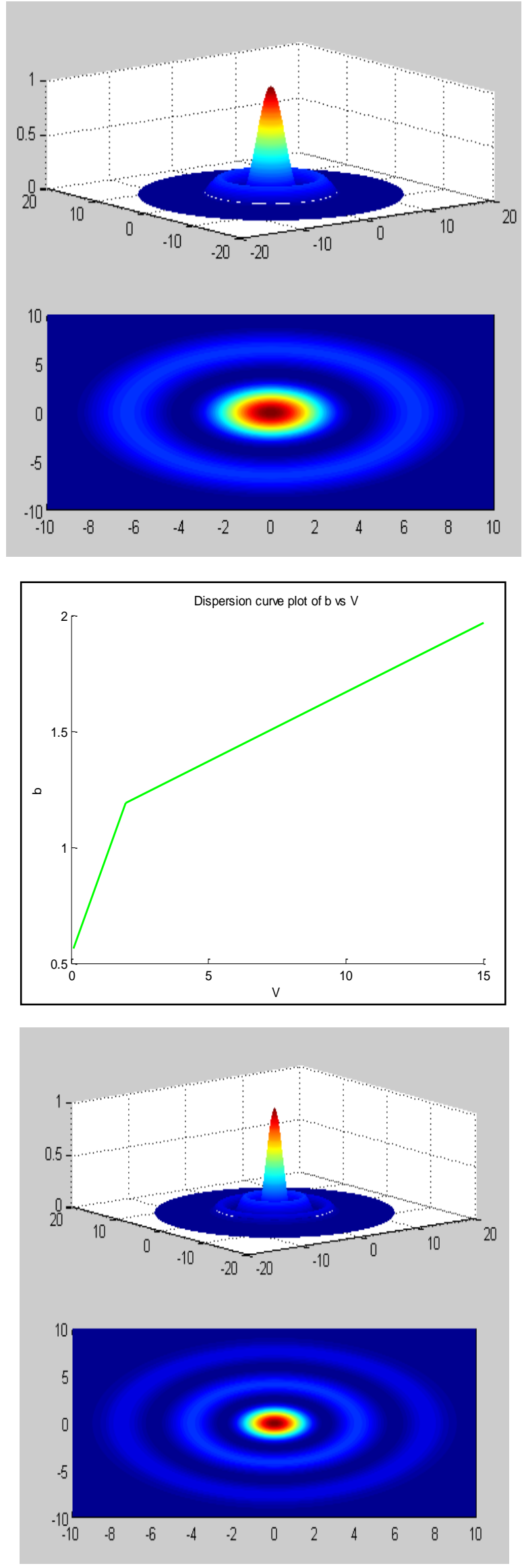

Fig-2 Fundamental field (LP) distribution of Bragg fiber 


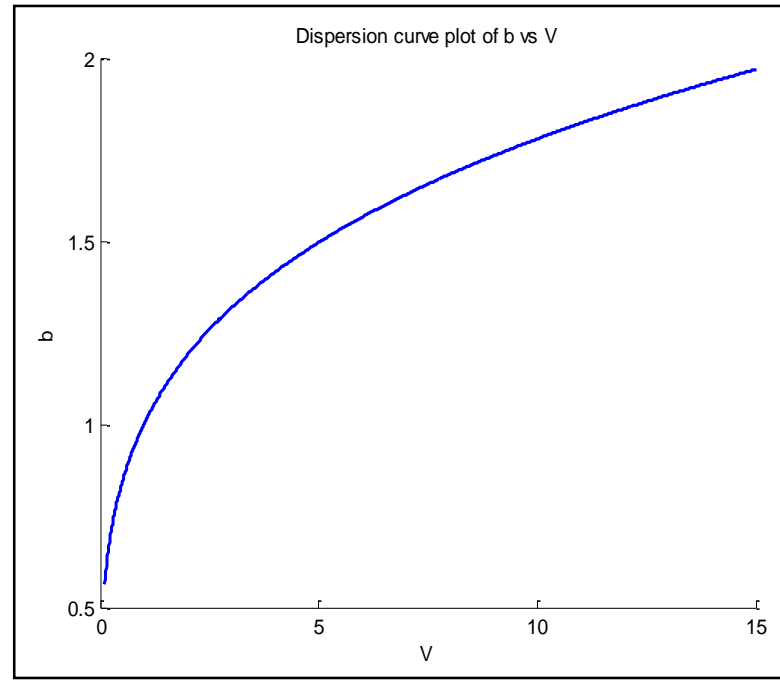

Fig-3 Variation of normalized propagation constant with respect to normalized frequency green for heterogeneous, blue for homogeneous mode.

\section{CONCLUSION}

In this paper, we study the propagation properties of high index core Bragg fiber. We have numerically explained the capability and tenability of the Bragg fiber, for some exact designs, we have found fundamental mode field solution of Bragg optical fiber and dispersion curve for the same in two different modes.

\section{REFERENCES}

[1] Yeh, P., Yariv, A., \& Marom, E. (1978). Theory of Bragg fiber. JOSA, 68(9), 1196-1201.
[2] Fink, Y., Ripin, D. J., Fan, S., Chen, C., Joannopoulos, J. D., \& Thomas, E. L. (1999). Guiding optical light in air using an all-dielectric structure. Journal of Lightwave Technology, 17(11), 2039.

[3] Ibanescu, M., Fink, Y., Fan, S., Thomas, E. L., \& Joannopoulos, J. D. (2000). An all-dielectric coaxial waveguide. Science, 289(5478), 415-419.

[4] Y. Xu, R. K. Lee, and A. Yariv, "Asymptotic analysis of Bragg fibers,"Opt. Lett., vol. 25, pp. 1756-1758, (2000).

[5] Ouyang, G., Xu, Y., \& Yariv, A. (2001). Comparative study of air-core and coaxial Bragg fibers: single-mode transmission and dispersion characteristics. Optics Express, 9(13), 733-747.

[6] Xu, Y., Ouyang, G. X., Lee, R. K., \& Yariv, A. (2002). Asymptotic matrix theory of Bragg fibers. Journal of lightwave technology, 20(3), 428.

[7] Ouyang, G., Xu, Y., \& Yariv, A. (2002). Theoretical study on dispersion compensation in air-core Bragg fibers. Optics express, 10(17), 899-908.

[8] Yeh, P., \& Yariv, A. (1976). Bragg reflection waveguides. Optics Communications, 19(3), 427-430.

[9] Chatterjee, S., Mondal, K., Khan, S. N., \& Chaudhuri, P. R. (2012, December). Exact mode field solutions and dispersion characteristics of $\mathrm{N}$-layered high-index-core Bragg fiber. In International Conference on Fibre Optics and Photonics (pp. M1A-6). Optical Society of America.

[10] Guo, S., Albin, S., \& Rogowski, R. (2004). Comparative analysis of Bragg fibers. Optics express, 12(1), 198-207. 